\title{
Gastric Bleeding with Multiple Organs Involvement of Eosinophilic Infiltration: A Case Report and Literature Review
}

\author{
Vinay-Pratap Singh ${ }^{1}$,Hong-Lin $\mathrm{Wu}^{1}$,Xiao-Feng $\mathrm{Ye}^{2}$, Zheng-Xing Jiang ${ }^{1}$, \\ Yuan $\mathrm{Shi}^{3}$ \\ ${ }^{1}$ (Department of Radiology, the Affiliated Wujin Hospital of Jiangsu University, Changzhou, 213002, \\ Jiangsu, China) \\ ${ }_{2}^{2}$ (Department of Gastroenterology, the Affiliated Wujin Hospital of Jiangsu University, Changzhou, \\ 213002, Jiangsu, China) \\ ${ }^{3}$ (Laboratory of Hematology, the First People's Hospital of Changzhou City; Changzhou, \\ 213003; Jiangsu, China)
}

\begin{abstract}
Eosinophilic infiltration (EI) is commonly causedbyparasitic infections, fungal infection, drug allergy, neoplastic disease, hyper eosinophilic, familial inheritance syndrome, inflammation etc. We report a case of 69 yrs oldman presented as upper digestive tract hemorrhage and abdominal distension for 10 days with eosinophilic infiltration involving liver, gastrointestinal tract (GIT) and lungs. For this case, contrast-enhanced CT of liver showed multiple small, oval or round and low attenuating nodules with well or fuzzy margins on sequential arterial and venous phase scans. Pulmonary CT showed small to medium sized multiple irregular nodule with surrounding airspace consolidation or ground-glass opacity and interstitial changes.Gastroendoscopyshowed diffuse bulky mucosa in the fundus and body of the stomach with erosion at surface, and an ulcer at gastric body. Eosinophils count was as high as $26.5 \times 10-9 / L(0.02-0.52 \times 10$ $9 / L$ ). The finally diagnose was made by bone biopsy.Treatment had been givenoral prednisone and he was followed-up long term with no evidence of recurrence.
\end{abstract}

Keywords:Eosinophilia, Liver, Lung, Gastrointestinal tract, Imaging, Haematemesis

\section{Core tip}

Eosinophilic infiltrations (EI) of multiple organs involving liver, gastric and lungs are very rare.Radiological and endoscopic examinations showed multiple nodules of different in various shape or size at liver, lungs and ulcers are seen at GIT. Eosinophils counts and bone marrow biopsy played a key role for final diagnosis of rare disease is EI of multiple organs. In order to gain a detailed understanding of this rare disease, we reviewed the literature and report here on a recent case of multiple organ eosinophilic infiltration disease in our hospital.

\section{Introduction}

Eosinophilic infiltration (EI) has been described as occurring in the skin, lungs, bones, tongue, urinary bladder, vulva, and nasopharynx. Liver, urinary bladder and gastrointestinal tract (GIT) involvements are very rare ${ }^{[1]}$. To the best of our knowledge, however, hepatic EI involving with GIT and lungs hasn't been reported. Here, we report a case of EI involving liver, GIT, and lungs, with gastric ulcer presented as haematemesis.

\section{Case report}

In July 2015, a 69year-old male patient was brought to our emergency department because he presented spitting blood for 6 hours, with abdominal distension and stomach-ache for 10 days. Sometimes symptoms presented nausea, vomiting and also some episodes of fever without chills. Acid reflux, belch, anorexia, diarrhea or cough and other signs were not presented. There was no history of allergy to any food or medicine.In physical examination, tenderness was present at upper abdomen. Abdominal Ultrasound showed no obvious abnormalities in liver, gallbladder, spleen, pancreas, etc. Gastroendoscopy showed diffuse bulky mucosa in the fundus and body of the stomach with erosion at surface. An ulcer, whose size about $2 \mathrm{~cm} \times 2.5 \mathrm{~cm}$, was also seen at gastric body, with mucosal congestion and edema around it. Gastric wall was stiffen and inflammation also seen at duodenal bulb (Fig.1 A, B). Then biopsy specimen was taken.Pulmonary CT showed multiple small to medium sized irregular nodules with surrounding airspace consolidation or ground-glass opacity, which involved mainly the marginal regions of the middle and lower lungs (Fig.2 A, B).Contrast-enhanced CT of liver showed multiple, small, round or oval and subtle hypo-attenuating lesions with well or fuzzy margins(Fig.3 A, B).Immunohistochemistry report revealed CA-125 as $53.2 \mathrm{U} / \mathrm{ml}(0-35 \mathrm{U} / \mathrm{ml})$, which was high. Hemogram showed white blood cell count as high as $37.11 \times 10^{-9} / \mathrm{L},\left(3.5-9.5 \times 10^{-9} / \mathrm{L}\right)$, neutrophils count $24.1 \times 10^{-9} / \mathrm{L}(1.8-$ 
$\left.6.3 \times 10^{-9} / \mathrm{L}\right)$, eosinophils ratio $71.4 \%(0.4-8 \%)$, and eosinophils count $26.5 \times 10^{-9} / \mathrm{L} \quad\left(0.02-0.52 \times 10^{-9} / \mathrm{L}\right)$. Histopathological specimen report of gastric biopsy showed inflammatory necrosis and granulation.

Bone biopsy was also done. In bone marrow aspiration, marrow aspirate smear showed large and round eosinophil granulocytes (Fig. 4. After a complete diagnostic work-up, EI with multiple organs involvement was diagnosed.Treatment had been included the use of steroidal medication, and after several months of oral prednisone treatment, the eosinophils ratio became normal. We followed-up the patient for a long term. After one year, we performed a following CT of chest and abdomen. CT images showed that the lesions had nearly all resolved in lungs and liver (Fig.5 A, B).
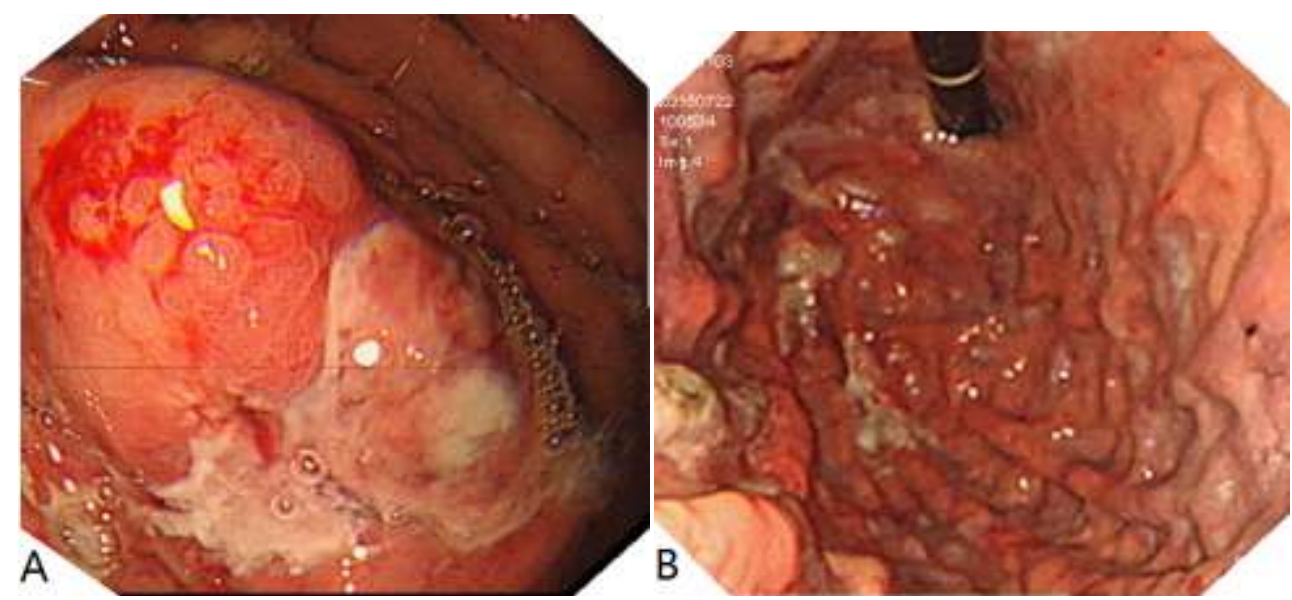

Fig. 1. Endoscopic photographs of eosinophilic gastroenteritis. A, A $2 \mathrm{~cm} \times 2.5 \mathrm{~cm}$ ulcer on gastric notch has whitish base, with mucosal congestion and edema around the ulcer. B,Gastroendoscopy showeddiffuse bulky mucosa.

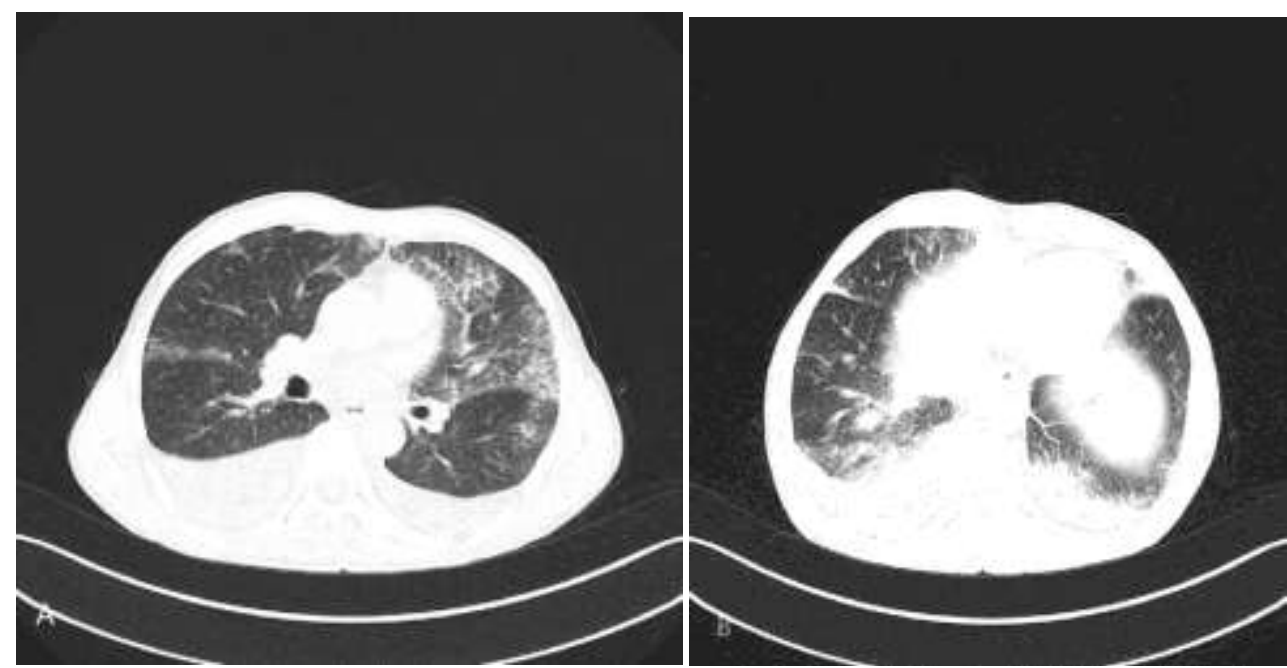

Fig 2.CTexamination of chest. A, CT showedinterstitial changeof the lung. B,Irregular nodulewith surrounding ground-glass opacity involved right inferior lobar. 

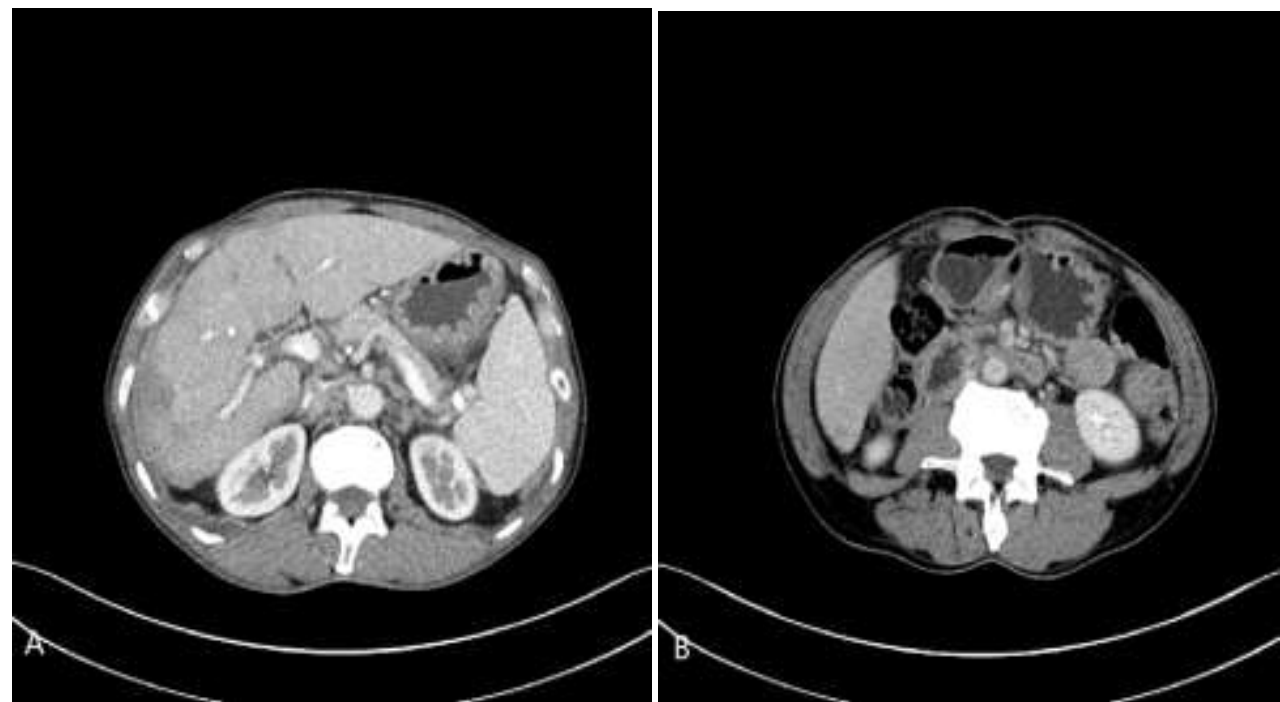

Fig 3. Enhanced CT examination of liver. A,CT scans showed patchy lesion in the right lobe of liver, more obvious in the arterial phase.B, Small, round, subtle hypo-attenuating lesionsinvenous phase.

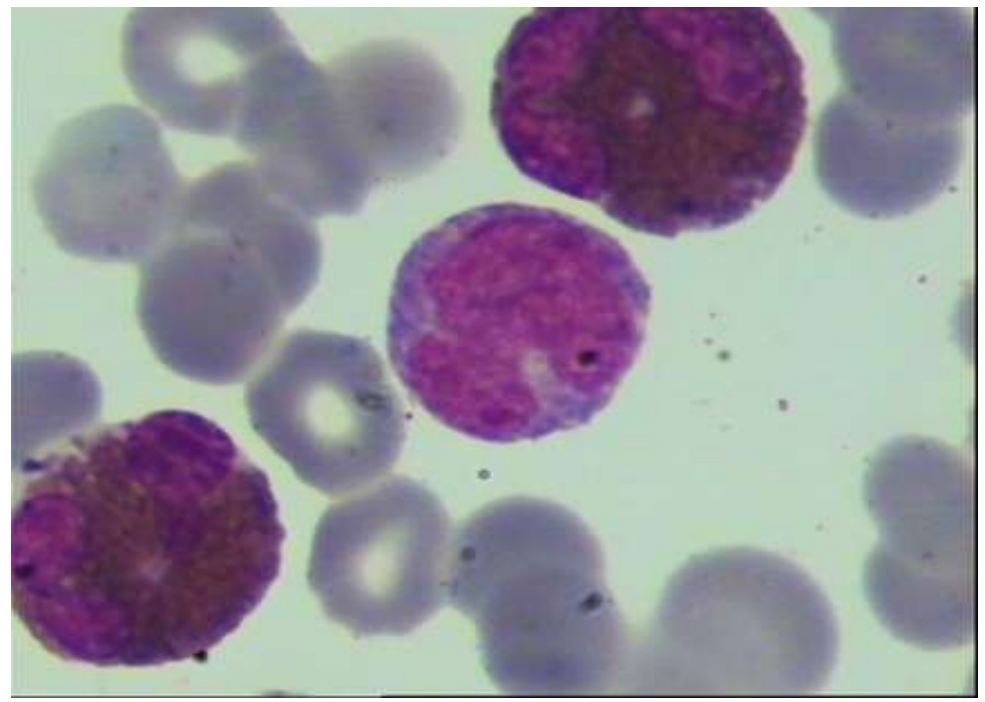

Fig 4. Marrow aspirate smear shows large and round eosinophil granulocytes with closely arranged orange eosinophilic granules throughout the cytoplasm(Wright stain, 1000×)

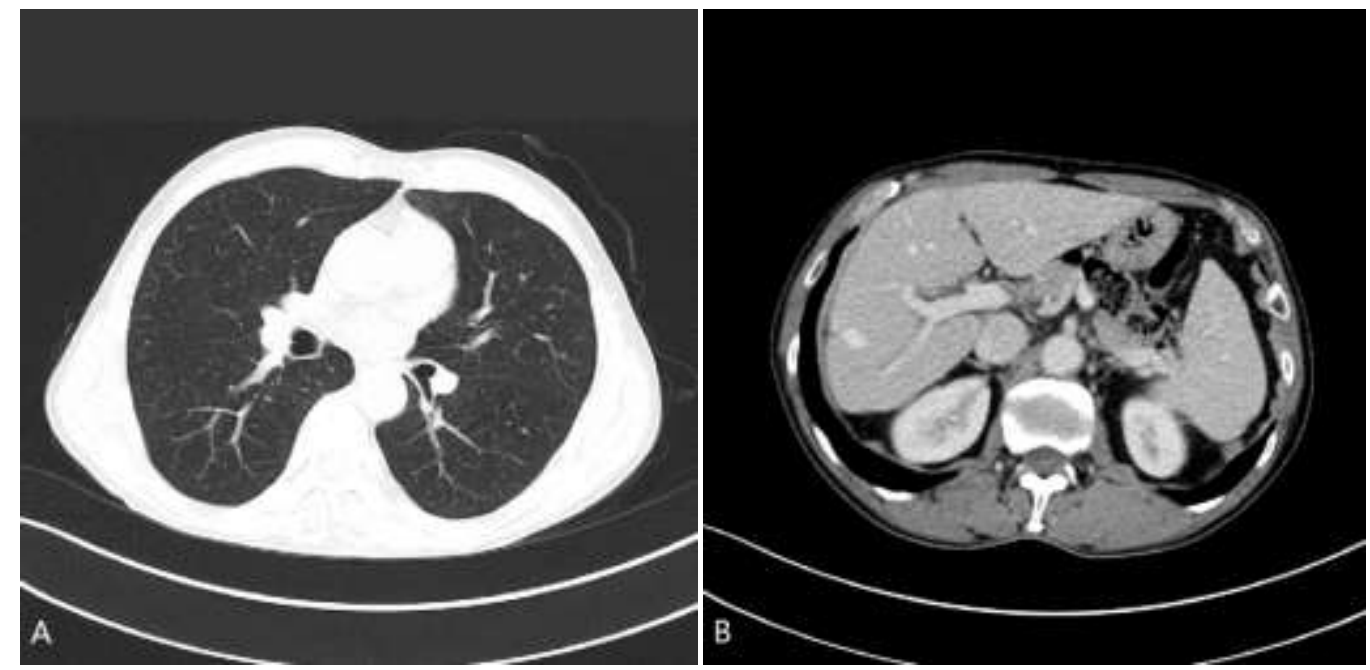

Fig 5. Follow up CT of lung and liver. A,The lesion of the lung has dissolved completely. B,The lesion of the liver has shrunk significantly. 


\section{Discussion}

EI may involve multiple organs like lungs, GIT, liver, gall bladder, urinary bladder, etc. The common causes of EI are parasitic infections, fungal infection, drug allergy, neoplastic disease, hyper eosinophilic, familial inheritance syndrome, inflammation, etc ${ }^{[2,3]}$. In our case, multiple organs of liver, GIT, and lungs were involved in EI.

Hepatic EI is also known as hepatic eosinophilia or eosinophilic liver abscess. It was first reported by Kaplan et al in $2001^{[4]}$. The typical CT examination findings are multiple, small $(<2 \mathrm{~cm})$, oval or round, subtle hypo-attenuating lesions with well or fuzzy margins, which are most prominent during the portal venous phase. The CT findings of hepatic EI always differentiate well from other infectious liver abscesses. Most of the hepatic EI are encountered incidentally in asymptomatic individuals with marginal eosinophils. Hepatic EI is mainly diagnosed clinically based on marginal eosinophils and imaging findings.

Eosinophilic gastrointestinal tract disorders are a group of eosinophilic diseases which include esophagitis, gastroenteritis, enteritis, and colitis. Eosinophilic gastroenteritis is the rarest from among these disorders and heterogenous disease characterised by EI of the GIT ${ }^{[5]}$. EI of GIT was first described in 1937.In 1961, Ureles et al systematically synthesized and categorized the cases of eosinophil cell infiltration in GIT and described its clinical characteristics of GIT extensively ${ }^{[6]}$.Endoscopy is useful to make diagnosis. Sometimes diffuse inflammation results in involvement of multiple layers, complete loss of villi, fibrosis and submucosal oedema. Definitive Diagnosis contains histopathological cogent evidence of EI in biopsy specimen slides. Microscopy usually shows more than 20 eosinophils per high power field. The lesions of EI are frequently patchy, and biopsy can sometimes be missed. In such circumstances, full thickness specimen may be needed by laparoscopy. In our case, although gastroendoscopy showed diffuse bulky mucosa in the fundus and body of the stomach, and an ulcer was also seen at gastric body, histopathological specimen didn't show the EI, only showing inflammatory necrosis and granulation. The negative pathologic result of our gastroendoscopy might be that we didn't get deep enough biopsy.

EI of the lung is characterized by EI in the lungs, along with radiologic abnormalities, peripheral blood eosinophilia and impaired lung function. In the recent literature, several different terms have been used to describe the pulmonary lesion. So far, EI of the lungs and pulmonary EI are the most commonly used. EI of lung was firstly described in 1951 by Farinacci ${ }^{[7]}$. He reported two cases diagnosed by lung biopsy.CT showed small to medium sized multiple irregular nodule with surrounding airspace consolidation or ground-glass opacity involving mainly the marginal regions of the upper and middle lungs. Most Commonly EI of lungs are misdiagnosed as interstitial pulmonary fibrosis or emphysema but rarely mistaken with malignancy or bronchiolitis obliterans organising pneumonia ${ }^{[8]}$. EI of lungs are associated with bone but rarely associated with liver and $\mathrm{GIT}^{[3,6]}$. For our case, we made the diagnosis according to imaging appearance and follow-up without lung biopsy.

From what is mentioned above,clinical diagnosis of EI is usually based on a combination of clinical symptoms, laboratory abnormalities, and imaging findings after exclusion of common identifiable causes. Multi organs involvement of EI can be overlooked radiologically and histopathologically. Therapy for EI involving liver, GIT and lungs hasn't been standardized. Treatment has been included the use of antibiotics, steroidal and non-steroidal, antihistamines and doxorubicin ${ }^{[9]}$. In our case, we gave oral prednisone for several months. This patient has been followed up for more than 1 year. The eosinophil ratio gradually become normal and a repeated $\mathrm{CT}$ of chest and abdomen showed no obvious abnormality anymore. The patient has remained disease-free and asymptomatic for several months till now.

\section{Conclusion}

We report a rare case of multiple organs involvement of EI. Moreover, toour knowledge, this is the first case of EI involving liver, GIT, and lungs. We also wish to highlight that liver Elis easily misdiagnosed as abscess or necrosis, andlung EI as interstitial pulmonary fibrosis or emphysema in clinical or imaging presentations.

\section{Acknowledgements}

Written informed consent was obtained from the patient for publication of this case report and any accompanying images.

\section{Funding}

Not applicable.

\section{Availability of data and materials}

Not applicable. 


\section{Authors' contributions}

All authors contributed equally in writing and reviewing work.

\section{Competing interests}

The authors declare that they have no conflicts of interests.

\section{Conflict of interest}

On behalf of all authors, the corresponding author states that there is no conflict of interest regarding this study.

\section{References}

[1]. Kim MS, Park H, Park CS, Lee EJ, Rho MH, Park NH, et al. Eosinophilic cystitis associated with eosinophilic enterocolitis: case reports and review of the literature. Br J Radiol2010; 83(990):122-5. [PMID:20505026 DOI: 10.1259/bjr/36109223]

[2]. Johkoh T, Müller NL, Akira M, Ichikado K, Suga M, Ando M, et al. Eosinophilic lung diseases: diagnostic accuracy of thin-section CT in 111 patients. Radiology2000; 216(3): 773-80. [PMID: 10966710 DOI:10.1148/radiology.216.3.r00se01773]

[3]. Rivera LS, Rivera IF, Toro DH, Gutierrez J, Acosta E. Eosinophilic Liver Infiltration:case report. ACG Case Rep J2015; 3(1):63-65. [PMID: 26504883 doi:10.14309/crj.2015.102]

[4]. Kaplan KJ, Goodman ZD, Ishak KG. Eosinophilic granuloma of the liver: a characteristic lesion with relationship to visceral larva migrans. Am J Surg Pathol2001; 25(10):1316-21. [PMID: 11688468]

[5]. Zhang MM, Li YQ. Eosinophilic gastroenteritis: A state-of-the-art review. J Gastroenterol Hepatol2016; 2. [PMID: 27253425 DOI: 10.1111/jgh.13463]

[6]. Ureles AL, Alschibaja T, Lodico D, Stabins SJ. Idiopathic eosinophilic infiltration of the gastrointestinal tract, diffuse and circumscribed; a proposed classification and review of the literature, with two additional cases. Am J Med1961; 30:899-909. [PMID: 13779299]

[7]. Farinacci CJ, Jeffrey HC, Lackey RW. Eosinophilic granuloma of the lung; report of two cases. U S Armed Forces Med J1951; 2(7):1085-93. [PMID: 14855860]

[8]. Kim SJ, Bista AB, Park KJ,Kang DK, Park JH, Park KJ, et al. Simple pulmonary eosinophilia found on follow-up computed tomography of oncologic patients.Eur J Radiol2014; 83(10):1977-82. [PMID: 25082475 DOI:10.1016/j.ejrad.2014.07.004]

[9]. Chen MJ, Chu CH, Lin SC, Shih SC, Wang TE. Eosinophilic gastroenteritis: clinical experience with 15 patients. World J Gastroenterol2003; 9(12): 2813-6. [PMID: 14669340] 